\title{
The spectroscopy of singlets and triplets excites electronic states, spatial and electronic structure of hydrocarbons and quantum classifications in chemmotology
}

\author{
A.E. Obukhov* \\ 25th State Research Institute of Chemmotology of the Ministry of Defense, 121467 Moscow, Russia

\begin{abstract}
In this work we demonstrate the physical foundations of the spectroscopy of the grounds states: E- and X-ray, (RR) Raman scattering the NMR ${ }^{1} \mathrm{H}$ and ${ }^{13} \mathrm{C}$ and IR-, EPR- absorption and the singlets and triplets electronic excited states in the multinuclear hydrocarbons in chemmotology. The parameters of UV-absorption, RR-Raman scattering of light, the fluorescence and the phosphorescence and day-lasers at the pumping laser and lamp, OLEDs and OTETs- are measurements. The spectral-energy properties are briefly studied. The quantum-chemical LCAO-MO SCF expanded-CI PPP/S and INDO/S methods in the electronic and spatial structure hidrocarbons are considered.
\end{abstract}

In this work the modern views on the nature of the conversion processes are outlined, and the variation of their rate with the type of transition and with the energy spectrum and structure of the molecule are analyzed. The relationship between spectra characteristics and phenomena of the scattering, absorption and reabsorptions, luminescence, emissions and generation and the structure of hydrocarbons molecules are discussed theoretically. The full spectrums of excited singlets and triplets electronic states (STElExSt) of the quantumchemical LCAO-MO SCF-CI INDO/S methods were calculated in [1] (Fig. 1). Under deactivations energy of pumping in STElExSt it is transformed to optics (fluorescence (Fl), phosphorescence $(\mathrm{Ph})$ ) and nonradiative transitions. The spin-orbitals interactions involved in STElExSt and the effect of spatial and electronic structure and other factors are considered. The hydrocarbons are given into the five types according to their physics of phenomena and a justification of this classification. The absolute quantum yield of fluorescence $(\mathrm{QyFl})$ and phosphorescence $(\mathrm{QyPh})$, and the rate constant $(\mathrm{Rc})$ intercombinations (IntCc) and $\mathrm{Rc}$ innercombinations convertions ( $\mathrm{InCc}$ ) between the lowest and highly STElExSt are offered as

$$
\begin{gathered}
\gamma_{f l}=k_{S_{1}^{*} \rightarrow S_{0}}\left(k_{S_{1}^{*} \rightarrow S_{0}}+k_{S_{i}^{*} \approx S_{0}}+\sum_{i=1, j=1}^{n, m} k_{S_{i}^{*} \ll T_{j}}\right), \\
\gamma_{p h}=k_{T_{1} \rightarrow S_{0}} \sum_{i=1, j=1}^{n, m} k_{S_{i}^{*} \ll T_{j}}\left(k_{S_{1}^{*} \rightarrow S_{0}}+k_{S_{1}^{*} \approx S_{0}}+\sum_{i=1}^{n} k_{S_{1}^{*} \approx T_{m}}\right)\left(k_{T_{1} \rightarrow S_{0}}+k_{T_{1} \approx S_{0}}+k^{*}\left(T_{1} \rightarrow T_{j}\right)\right),
\end{gathered}
$$

\footnotetext{
*Corresponding author: aobukhov@fo.gpi.ru
} 
where ${ }^{k} S_{1}^{*} \rightarrow S_{0}$ and ${ }^{k} T_{1} \rightarrow S_{0}$ are the RcFl and $\mathrm{RcPh}$, respectively, $\sum_{i=1, j=1}^{n, m}{ }^{k} S_{i}^{*} \ll T_{j}$ is sum the rate constants (RC) of ItCc between all types of STElExSt, ${ }^{*}\left(T_{1} \rightarrow T_{j}\right)$ is the new entered empirical constant, which on efficiency of photoionization $\left(\boldsymbol{I}_{1}{ }^{*}\right),{ }^{k} S_{1}^{*} \approx S_{0}$ and ${ }^{k} T_{1} \approx S_{0}$ is the $\mathrm{RC}$ InC and RC ItCc from lowest the STElExSt.

(a)

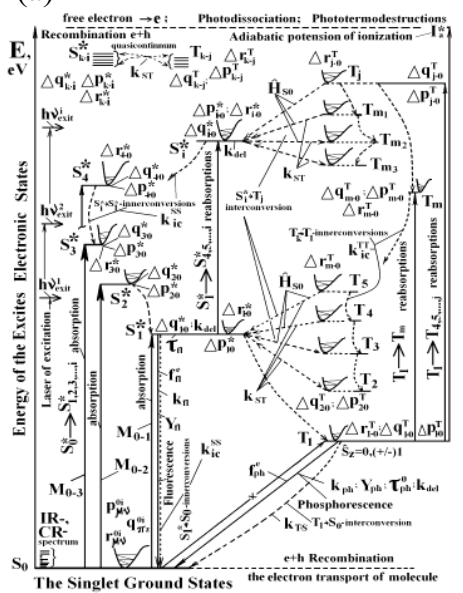

(b)

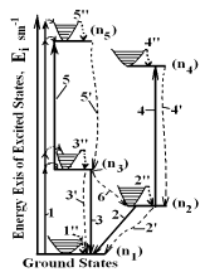

(c)

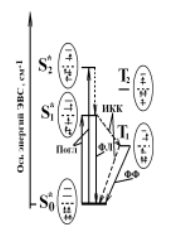

(d)

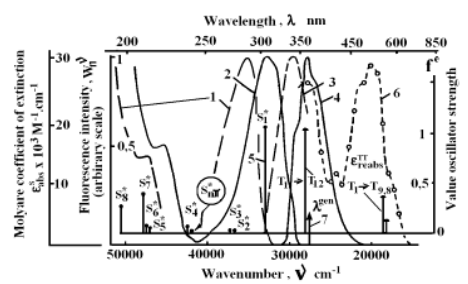

(e)

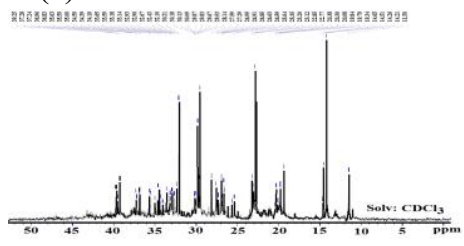

Fig. 1. (a). The full spectra of the optical and nonradiated transition between of the systems of the GrSt and between the singlets $\left(S^{*}{ }_{1, \ldots, \mathrm{i}}\right)$ and triplets $\left(T_{1, \ldots, \mathrm{j}}\right)$ ElExSt (the ion is the quartets $\left(Q_{\mathrm{n}}\right)$ and the duplets $D_{\mathrm{m}}$ ) with the LCAO-MO SCF expansion-CI INDO/S methods of calculations. According to the rules of selection on the orbital symmetry on the $S *_{1-}>S *_{2, \ldots, \text { i }}$ of singlet-singlets transitions. At the $\operatorname{ItCC}\left(S^{*}{ }_{1, \ldots, i}<\approx T_{\mathrm{f}}^{\alpha}\right)$ with intermediate the height triplets $T_{\mathrm{j}}$ states from the RC of ItCC is $k_{\mathrm{sT}} \approx$ $\left(10^{8} \div 10^{13}\right) \mathrm{s}^{-1}$ effectively population the lowest $S^{*}{ }_{1}$ and $T_{1}$ ElExSt. According to the rules of selection on the orbital symmetry on the $\mathrm{T}_{1}->\mathrm{T}_{1, \ldots, \mathrm{j}}$ of triplet-triplets transitions by populations the height $T_{\mathrm{j}}$ ElExSt it is impossible to identify with before ionizations the quasy-continuum of Rydberg states. The discrete mechanism of the multistage photoionization and photo-termo-destructions of hydrocarbons. Here $\Delta r_{\mu v}$ and $\Delta p_{\mu v}, \Delta q_{\mu v}$, and $\Delta \rho_{\mu v}$ - length and the stretching vibrations of bonds, change and full electronic density from atoms; (b) The five-levels of system the ElExSt. 1 is the energy of lasers pumping, 2 and 2' is the phosphorescence $(p h)$ and nonradiated transitions, 3 is of the fluorescence $(f l)$ and 3 ' nonradiated transitions, 4 is the singlet-singlet and 5 is triplet-triplet reabsorption (TTR) of electron-vibrations transitions (2',3',4' and $\left.5^{\prime}\right)$ the InCC of transitions, $(1,2, ", 3 ", 4$ ", 5 "') is the electron-vibrations conversion of transitions (picosecond relaxation), 6 is the InCC of transitions. Here $\left(n=n_{1}+n_{2}+n_{3}+n_{4}+n_{5}\right)$; (c) tree-levels of systems Yablonskii; (d) The spectra of UV-absorption $\left(\varepsilon^{v}\right.$ abs, 1,2$)$ and the fluorescence $\left(\mathrm{W}^{v} \mathrm{fl}, 3,4\right)$, and maximum the generation radiation of light $\left(\lambda^{\left.\max _{\text {gen }}, 7\right)}\right)$, and induced pumping the spectra of TTR $\left(\varepsilon^{\mathrm{TT}}\right.$ reabs, 5,6$)$ in the vapor $(2,3,5)$ and the solutions $(1,4,6,7)$. Vertical continuous lines is values of oscillator strength $\left(f^{\mathrm{f}} \mathrm{fl}\right.$,phosf and $f^{\mathrm{e}}$ reabs) on frequencies $\left(v^{\mathrm{ij}}\right)$ of the electronic $\mathrm{S}_{0-}>\mathrm{S} *_{1, \ldots, \mathrm{i}}$, and $\mathrm{T}_{1-}>\mathrm{T}_{1, \ldots, \mathrm{j}}$ transitions $\pi \pi^{*}$ - and $n \pi^{*}$ types and the values of designed by the LCAO-MO SCF-CI INDO/S for the lasers-active 2-phenyl1,3-oxazole (POP); (e) - dope fuel of Haitec-580.

Unusual behavior of the dysprosium $\mathrm{CF}$ energies in $\mathrm{Dy}_{2} \mathrm{BaNiO}$ was detected in the temperature range lower than $\mathrm{T}_{\mathrm{N}}$. All CF levels experience a shift (up to $10 \mathrm{~cm}^{-1}$ ) from their positions at $\mathrm{T}_{\mathrm{N}}$ (see, e.g., Fig. 1). Such behavior can be explained as being due to the spontaneous electric polarization that appears in the temperature range discussed [8]. Redistribution of the charge density influences the crystal field acting on dysprosium and leads to a renormalization of CF self-energies.

\section{References}

1. A.E. Obukhov, Spectroscopy of the ground and excited states of multinuclear compounds in different conditions (Satellite+, Moscow, 2010) 